\title{
NGF promotes synovial nerve sprouting
}

New data published in Arthritis \& Rheumatism suggest that extensive nerve remodeling in the synovium might contribute to arthritic joint pain. "While others have previously shown glimpses of this remodeling-one nerve fiber here or there-it was difficult for clinicians or basic scientists to understand or appreciate the extent and context of this reorganization," states Patrick Mantyh, of the University of Arizona, who led this study; "once one sees the extent of the reorganization, the clinical and therapeutic implications will be understood."

In a mouse model of inflammatory arthritis, Mantyh and colleagues demonstrated a marked increase in the density of both sensory and sympathetic nerves in regions of the knee joint synovium that were poorly or noninnervated in control animals. The newly sprouted nerve fibers occur in areas of the joint that are subject to considerable stress, and thus might be responsible for disease-associated pain-related behaviors observed in this model.

Mantyh and co-workers also found that early and sustained treatment (every 5 days after initiation of arthritis) with a blocking antibody targeting nerve growth factor (NGF) substantially reversed the ectopic nerve growth. Correspondingly, use and weight bearing of the affected limb improved by up to $50 \%$ and $75 \%$, respectively, compared with controls.

Interestingly, however, administration of anti-NGF antibodies did not affect neovascularization or inflammation in the injured joints. "These data may in part explain why there is frequently a disconnect between the degree of skeletal
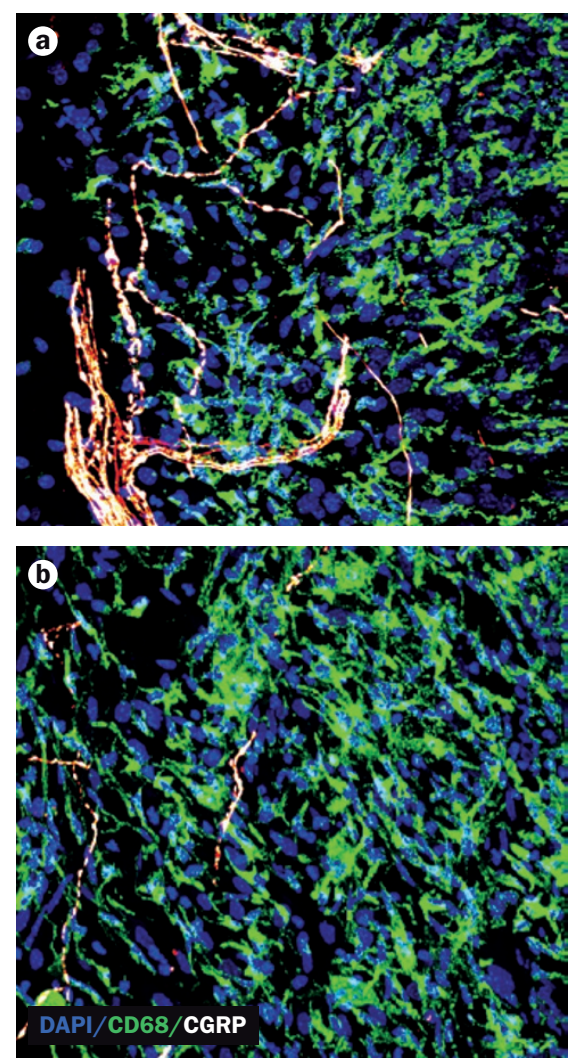

Ectopic remodeling of CGRP-expressing nerves in the inflamed synovium (a) is suppressed by NGF-blockade (b). Image courtesy of P. W. Mantyh.

injury and pain," says Mantyh. "Severity of chronic skeletal pain may be due as much to extent of the ectopic nerve reorganization as it is to the degree of the underlying skeletal disease or injury."

David Killock

Original article Ghilardi, J. R. et al. Neuroplasticity of sensory and sympathetic nerve fibers in the painful arthritic joint. Arthritis Rheum. doi:10.1002/art.34385 